\title{
UPON BULK CAVITATION APPEARING IN UNDERWATER EXPLOSIONS
}

\author{
Vasile NĂSTĂSESCU*, Ghiță BÂRSAN*** \\ "Military Technical Academy, Bucharest, Romania \\ ** "Nicolae Bălcescu" Land Forces Academy, Sibiu, Romania \\ nastasescuv@gmail.com, ghbarsan@gmail.com
}

\begin{abstract}
This paper presents some results of the author's researching in connection with SPH (smoothed particle hydrodynamics) method and underwater explosion numerical modelling. All about cavitation fundamentals are considered known and about cavitation effects upon the structures. The authors, deeply preoccupied in using of SPH method, as well in modelling of the underwater explosion effects upon structures, had to take into consideration the bulk cavitation. A main issue in this study was the knowing of the bulk cavitation domain and its characteristic parameters. Such researching was possible to be successfully carried out, only by using of the SPH method. Finally, the paper presents the relations and the working way for knowing of the bulk cavitation domain and also a numerical model using SPH method is presented. The numerical example regarding shape and dimensions of the bulk cavitation is presented together putting in evidence of some parameters which can make damages upon a structure that is in the bulk cavitation area.
\end{abstract}

\section{Keywords: bulk cavitation, shock wave, SPH method, underwater explosion}

\section{Introduction}

An underwater explosion is a very complex phenomenon which is very difficult regardless any approaching way. An underwater explosion is that explosion having the point of detonation below the water surface [4]. There are two types of underwater explosions: shallow and deep explosions. These types of underwater explosion can be categorized by empirical criterion [5], defined by the relation between $d$ (the explosion depth, in $\mathrm{ft}$ ) and $W$ (explosive mass, in pounds), so: if $d / \sqrt[3]{W}<1$ this means a shallow explosion and if $d / \sqrt[3]{W}>16$ this means a deep explosion.

What makes the difference between these explosion types is what means the explosion parameters and explosion effects.
But there was something in common: the phenomenon of cavitation.

An underwater explosion consists in converting of the charge instantly into hot gas (approximately $3000^{\circ} \mathrm{C}$ ) and in appearing of a shock pressure of up to over $5000 \mathrm{MPa}$. The shock wave propagates spherically into the water, having duration of $10^{-5} \ldots 10^{-3}$ seconds.

Next to other aspects regarding the underwater explosion, the cavitation is a very important one by its effects upon immersed structures. The phenomenon of cavitation appears when, in a water domain, a negative absolute pressure occurs.

A negative pressure leads to the tensile forces in the water, but this cannot sustain such forces and cavitation or separation is formed. Two types of cavitation exist: local cavitation and bulk cavitation. This occurs 
at the free surface, in a large area; the local cavitation occurs in a small area just at the fluid-structure interface. Both cavitation types have strong effects upon immersed structures. Other classification makes differences between contact explosion and non-contact explosion [9]. These types of underwater explosions are very important in evaluation of the explosion damage of a structure. But, no matter the explosion type (shallow or deep, contact or non-contact), cavitation phenomenon is present and this often has a significant contribution to damages.

\section{Underwater explosion parameters}

By explosion, a large quantity of energy is released and this is a function of the charge weight and of the stand-off distance.

The main parameters, like the peak pressure $P_{\max }$ in the shock wave front, the pressure profile $P(t)$ and the decay constant $\theta$ describing the exponential decay, can be calculated with formulas [1], [3]:

$$
\begin{aligned}
& P_{\max }=K_{1}\left(\frac{W^{\frac{1}{3}}}{R}\right)^{A_{1}} \\
& P(t)=P_{\max } \cdot e^{-\frac{t-t_{1}}{\theta}} \\
& \theta=K_{2} W^{\frac{1}{3}}\left(\frac{W^{\frac{1}{3}}}{R}\right)^{A_{2}}
\end{aligned}
$$

where $K_{1}, A_{1}, K_{2}$ and $A_{2}$ are constants depending on the explosive type. When the measure units of the international system are used, their values are presented in the Table 1.
Table 1. Constants of explosives

\begin{tabular}{|c||c|c|c|}
\hline \multicolumn{1}{|c||}{} & \multicolumn{3}{c|}{ Explosive Type } \\
\cline { 2 - 4 } & HBX-1 & TNT & PETN \\
\hline \hline$K_{1}$ & 20.42148 & 20.56589 & 22.47032 \\
\hline$A_{1}$ & 1.144 & 1.18 & 1.194 \\
\hline$K_{2}$ & 0.0734875 & 0.0761121 & 0.0682384 \\
\hline$A_{2}$ & -0.247 & -0.185 & -0.257 \\
\hline
\end{tabular}

The pressure results in $\mathrm{MPa}$ and exponential decay $\theta$ is calculated in milliseconds [ms]. The parameter $t$ (time) in the relation (2) is expressed in ms and represents the time elapsed from the detonation and respectively, the arrival time $t_{1}$ of the wave to a point (target). $R$ is the distance between explosion point and a considered point. These explosion parameters are only those which are used for calculus of the bulk cavitation area. Of course others parameters exist and they are used especially for explosion effects evaluation and for other issues evaluation.

\section{Bulk Cavitation Domain}

Bulk cavitation phenomenon appears as a result of the reflection of the shock wave at the free water surface. As we can see in the Figure 1, in a point (target) two wave front arrive: an incident one (compressive wave) and a reflected one (rarefaction wave).

Initially, incident wave travels in water with a velocity much faster than the sound speed, but rapidly this velocity goes down owing to temperature, salinity, pressure etc.; decreasing of the wave velocity does not go under sound speed in that water (aprox. $1500 \mathrm{~m} / \mathrm{s}$ ).

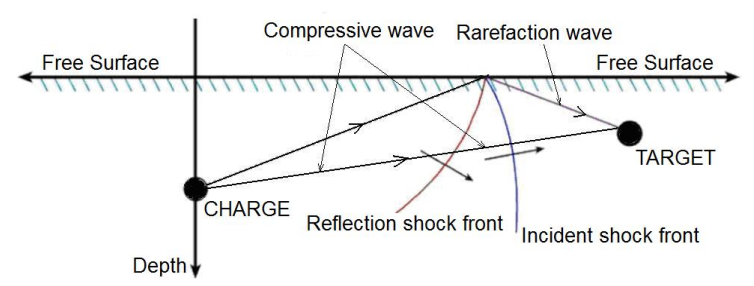

Fig. 1 Incident and reflected waves

At a moment, in a point, the summation of those waves becomes zero. Such a point is 
placed on the upper boundary of the bulk cavitation region (area, domain). Cavitation phenomenon occurs when the absolute pressure falls below the cavitation pressure, which is a negative pressure of $0.025 \mathrm{MPa}$, and the water can not support such resulting tensile load.

In the Figure 2, we can see the shock wave profiles of the pressure (incident) and rarefaction (reflected or image pressure) waves.

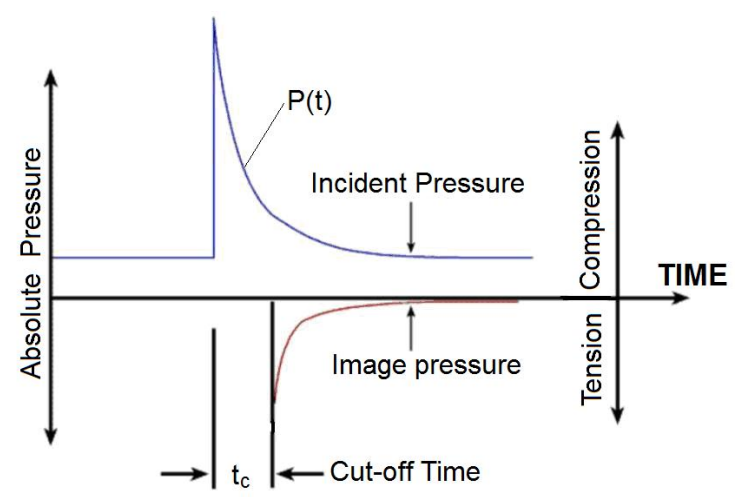

Fig. 2 Incident and image pressure

Image pressure profile is in fact the profile of the reflected wave considered coming like an incident pressure but from image point of the charge (Figure 4).

Because of symmetry, the space of cavitation is a 3D axisymmetric volume (around $\mathrm{Y}$ axis). In X-Y plane, we see only the cavitation area/domain (Figure 3 ).

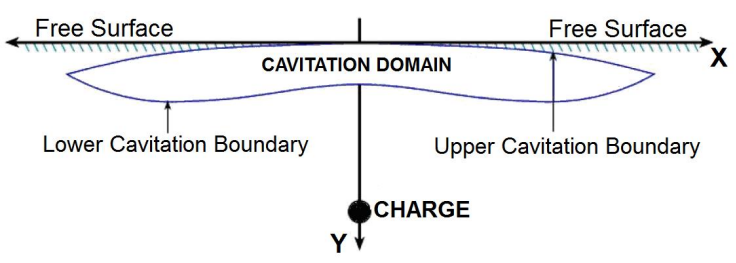

Fig. 3 Bulk cavitation domain

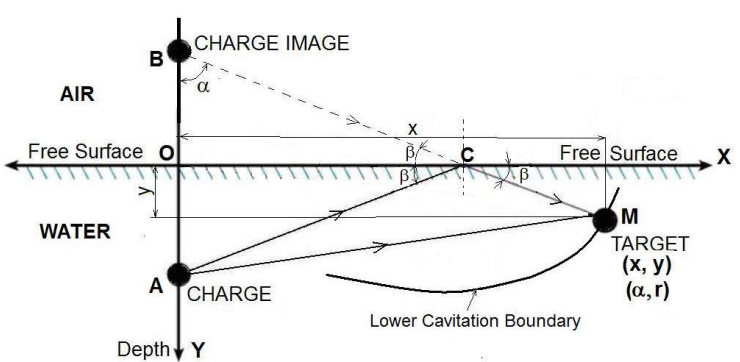

Fig. 4 The geometry of interference waves
Making the notations: $A M=r_{1} ; B M=r_{2}$; $O A=O B=D$, relations (4)...(6), can be written, where $\alpha$ and $r$ are the current polar coordinates. The $r$ coordinate takes the particulare value $r_{2}$ when $M$ stay just on the lower cavitation boundary.

$$
\begin{gathered}
\left\{\begin{array}{l}
\sin \alpha=\frac{x}{r}=s n \\
\cos \alpha=\frac{D+y}{r}=c s
\end{array}\right. \\
r_{1}=\sqrt{x^{2}+(D-y)^{2}}= \\
=\sqrt{r^{2}+4 D^{2}-4 D \cdot r \cdot c s} \\
r_{2}=\sqrt{x^{2}+(D+y)^{2}}=r
\end{gathered}
$$

The position of a point $\mathrm{M}$ (on the upper or on the lower cavitation boundary) can be expressed in cartezian $(x, y)$ or polar $(\alpha, r)$ coordinates. Using the relations (1)...(4) and the wave propagation speed in water $(C \approx 1500 \mathrm{~m} / \mathrm{s}$ ), we can write:

- incident (compressive) pressure:

$$
P_{i}=K_{1}\left(\frac{W^{\frac{1}{3}}}{r_{1}}\right)^{A_{1}} \cdot e^{-\frac{r-r_{1}}{C \cdot \theta}}=P_{i}(\alpha, r)
$$

- exponential decay or cut-off time, which represents the time when image pressure (Figure 2) has the same value of the incident pressure, but with negative sign:

$$
\theta=K_{2} W^{\frac{1}{3}}\left(\frac{W^{\frac{1}{3}}}{r_{1}}\right)^{A_{2}}=\theta(\alpha, r)
$$

- reflected (breaking) pressure, considered 
like an incident pressure coming from charge image:

$$
P_{r}=-K_{1}\left(\frac{W^{\frac{1}{3}}}{r}\right)^{A_{1}}=P(\alpha, r)
$$

- atmospheric pressure $P_{a}=101.353 \mathrm{kPa}$,

$$
P_{a}=\text { const }
$$

- hydrostatic pressure $\left(P_{h}\right)$,

$$
P_{h}=\rho \cdot g \cdot y=\gamma \cdot y=P_{h}(\alpha, r)
$$

where $\gamma$ is the specific gravity.

- Absolute pressure, in a point $(M)$, is:

$$
P_{a b s}=P_{i}+P_{a}+P_{h}
$$

- The total pressure, in a point ( $M)$, is:

$$
P_{t o t}=P_{a b s}+P_{r}=P_{t o t}(\alpha, r)
$$

The upper cavitation boundary is the locus of the points, where their coodinates fulfil the condition:

$$
P_{t o t}=P_{a b s}+P_{r}=0
$$

Practically, the relation (14) represents the upper cavitation boundary equation, having the form of relations (15).

$$
\begin{gathered}
K_{1}\left(\frac{W^{\frac{1}{3}}}{r_{1}}\right)^{A_{1}} \cdot e^{-\frac{r_{2}-r_{1}}{C \cdot \theta}}+P_{a}+\gamma \cdot y- \\
-K_{1}\left(\frac{W^{\frac{1}{3}}}{r_{2}}\right)^{A_{1}}=0
\end{gathered}
$$

The parameters $r_{1}$ and $r_{2}$ of the relation (15) can be easily expressed in Cartesian coordinates, using relations (5) and (6).

The reflected (breaking) pressure goes through the cavitation domain and goes on into uncavitated water beneath the lower boundary. Let's consider [2]. a spherical attenuation of the reflected pressure, in the form:

$$
P_{r}=P(\alpha, r)\left(\frac{r_{2}}{r}\right)^{A_{1}}
$$

Lower cavitation boundary comes from the require that the decay rates of those pressures (absolute and reflected) have to be the same, so that beyond the lower boundary no tension effect of the water to appear. Because of axisymmetric bulk cavitation domain, at a moment, we can take into account only the variable $r$; all what is true for a value of $r$, is true for all the values of $\alpha$. So, mathematicaly, we can write:

$$
\frac{d}{d r}\left(P_{r}\right)=\frac{d}{d r}\left(P_{a b s}\right)
$$

or,

$$
\frac{d}{d r}\left[P(\alpha, r)\left(\frac{r_{2}}{r}\right)^{A_{1}}\right]_{r=r_{2}}=\frac{d}{d r}[P(\alpha, r)]_{r=r_{2}}
$$

$\frac{d}{d r}\left[P(\alpha, r) \cdot r^{A_{1}}-P(\alpha, r) \cdot r_{2}^{A_{1}}\right]_{r=r_{2}}=0$

Noticing that the second term from left member of the relation (17) is a constant, for the considered limit, it remains that:

$$
\frac{d}{d r}\left[r^{A_{1}} \cdot P(\alpha, r)\right]_{r=r_{2}}=0
$$

where,

$$
r^{A_{1}} P(\alpha, r)=r^{A_{1}}\left[P_{i}(\alpha, r)+P_{a}+P_{h}(\alpha, r)\right]
$$


The connection between polar and cartesian coordinates, by relations (4)...(6); so, relation (8) and (19) get the form of relation (20) and that presented in the Figure 5.

$$
\theta=K_{2} W^{\frac{1}{3}}\left(\frac{W^{\frac{1}{3}}}{\sqrt{r^{2}+4 D^{2}-4 D \cdot r \cdot c s}}\right)^{A_{2}}
$$

$r^{A_{1}}\left\{\gamma \cdot c s+K_{1}\left(\frac{W^{\frac{1}{3}}}{\sqrt{4 D^{2}-4 \cdot c s \cdot D \cdot r+r^{2}}}\right)^{A_{1}} \cdot e^{-\frac{r-\sqrt{4 D^{2}-4 \cdot c s \cdot D \cdot r+r^{2}}}{C K_{2} W^{\frac{1}{3}}\left(\frac{W^{\frac{1}{3}}}{\sqrt{4 D^{2}-4 \cdot c s \cdot D \cdot r+r^{2}}}\right)^{A_{2}}}} \cdot\left[\frac{\frac{2 r-4 D \cdot c s}{2 \sqrt{4 D^{2}-4 \cdot c s \cdot D \cdot r+r^{2}}}-1}{\left.C K_{2} W^{\frac{1}{3}\left(\frac{W^{\frac{1}{3}}}{\sqrt{4 D^{2}-4 \cdot c s \cdot D \cdot r+r^{2}}}\right.}\right)^{A_{2}}}-\right.\right.$

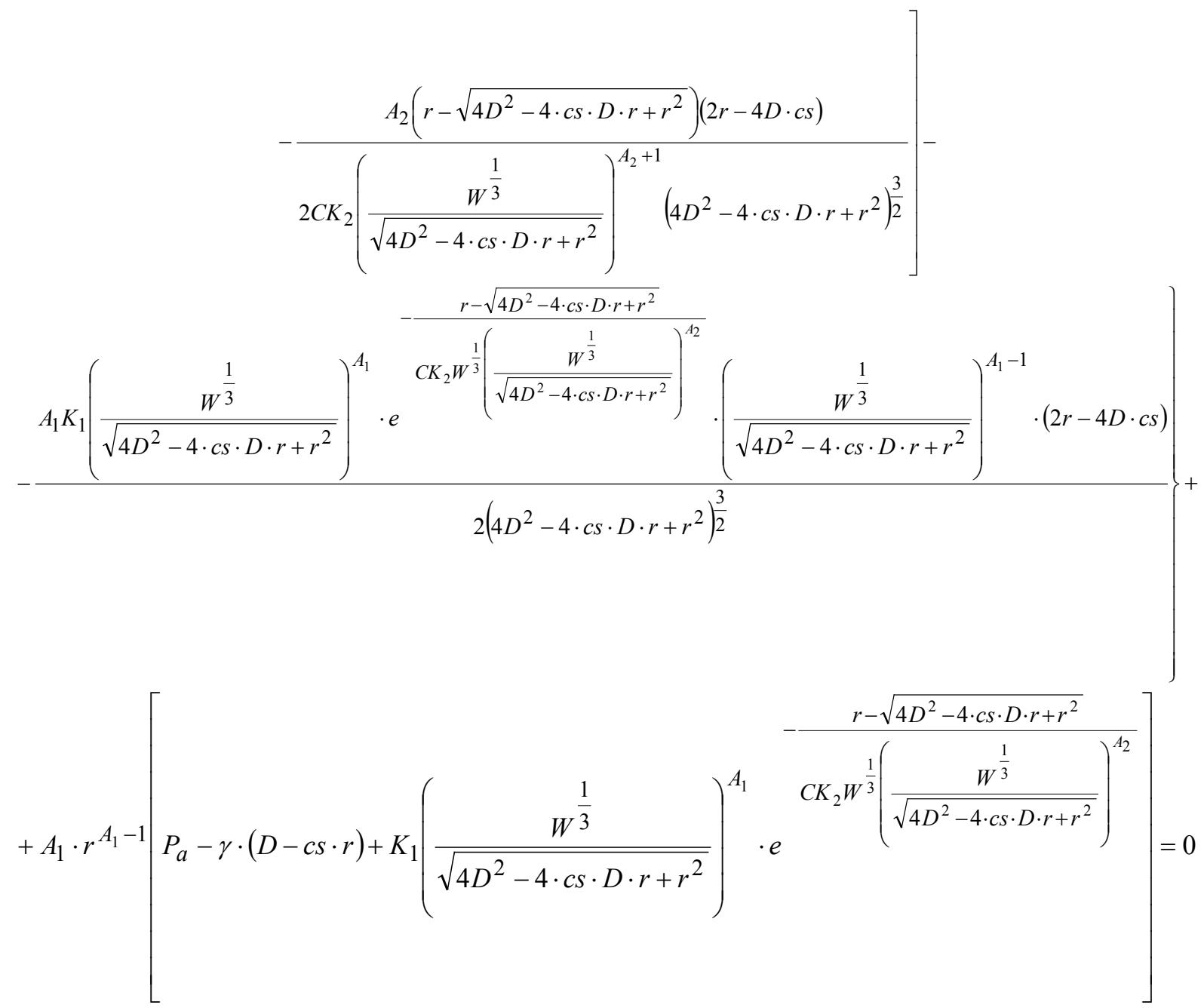

Fig. 5 Calculus details of the relation (19)

Taking into account that the parenthesis, of the relation presented in the Figure 5, has to be zero and making some substitutions for coming back to cartesian variable, we can write the relation (21). This relation represents the lower cavitation boundary equation.
This, together relations (5) and (6), gives us the cartesian coordinates of the points staying on this curve. The space sided by these curves is the cavitation domain (Figure 3). Those two curves intersect in a point. The coordinates of this point represent a solution of both curves. 


$$
\begin{gathered}
\frac{P_{i}}{C \theta}\left[\frac{r_{2}-2 D \frac{D+y}{r_{2}}}{r_{1}}\left(1-\frac{A_{2}\left(r_{2}-r_{1}\right)}{r_{1}}\right)-1\right]- \\
-\frac{A_{1} P_{i}}{r_{1}^{2}}\left(r_{2}-2 D \frac{D+y}{r_{2}}\right)+\gamma \frac{D+y}{r_{2}}+ \\
+\frac{A_{1}}{r_{2}}\left(P_{i}+P_{a}+\gamma \cdot y\right)=0
\end{gathered}
$$

Owing to symmetry, it is enough to determinate the upper and lower boundaries only for positive values of coordinates.

Practical calculus can be made by different available software or by an own software. In the Figure 6, the bulk cavitation area is presented ( $a$-only a half, $b$-whole xOy area), for the case of an underwater explosion having: explosive quantity $W=0.150 \mathrm{~kg}$, explosion depth $D=2.50 \mathrm{~m}$, atmospheric pressure $P_{a}=101 \mathrm{kPa}$, sound speed in water $C=1500 \mathrm{~m} / \mathrm{s}$ and specific gravity $\gamma=9810 \mathrm{~N} / \mathrm{m}^{3}$. The Figure 6-a shows both the shape and also the dimensions ( $L, M, N$ points) of the bulk cavitation area in the $\mathrm{xOy}$ plane. The 3D bulk cavitation domain is easy to imagine, being an axisymmetric volume around $\mathrm{Y}$ axis.

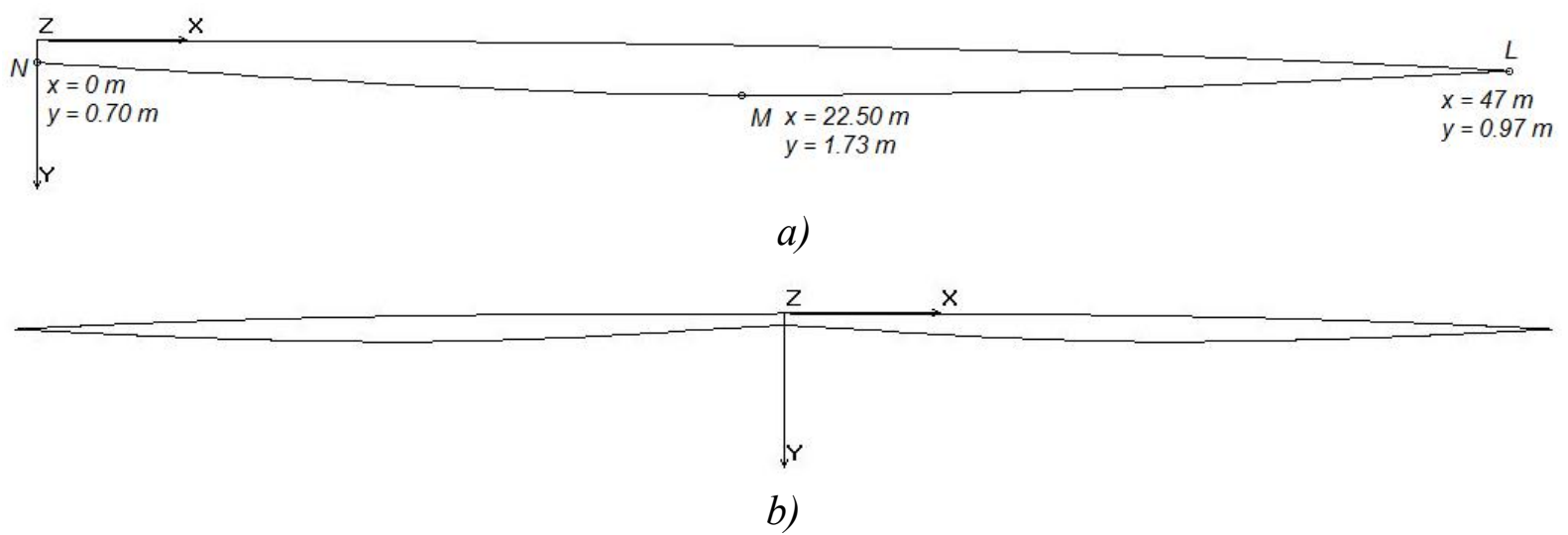

Fig. 6 Bulk cavitation area for the given case

\section{Numerical Example}

The numerical example, presented below, is based on using of the Smoothed Particle Hydrodynamics (SPH) Method [6], [7], [8]. For avoiding a long computational time, the cavitation phenomenon is simulated by a $2 \mathrm{D}$ axisymmetric model. The water domain has side dimensions $20 \mathrm{~m}$ along $\mathrm{x}$ direction and $5 \mathrm{~m}$ along y direction.

The geometrical and numerical models are presented in the Figure 7 (a-whole model, b-a a detail). The arrangement of all the particles is an uniform one, with a distance between nodes of $0.05 \mathrm{~m}$. So, the total particles number is 39996 and the TNT particles number is only 4 , as the Figure $7-b$ shows.
The bottom of the water space is considered a rigid wall.

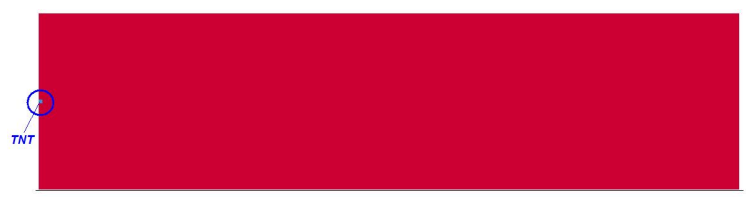

a)

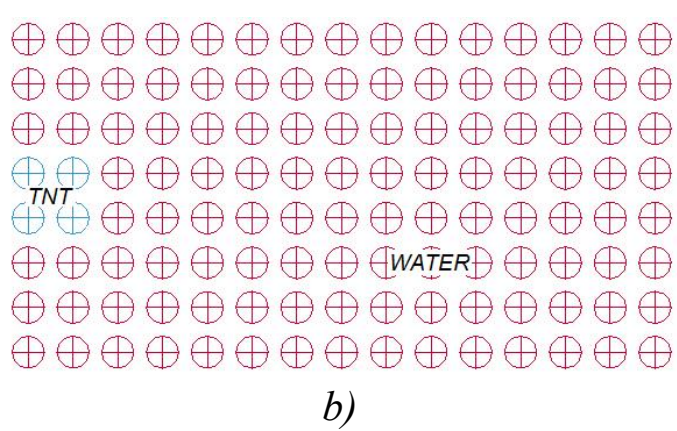

Fig. 7 SPH numerical model

For the water modelling, the elastic- plastic- 
hydro material model was used, together Gruneisen equation of state (EOS). For the TNT modelling, the high-explosive-burn material model was used, with JonesWilkens-Lee (JWL) equation of state.

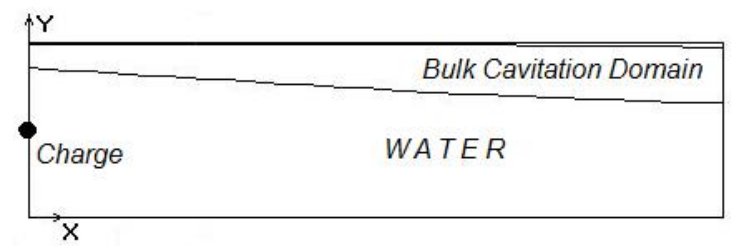

Fig. 8 Domain of numerical analysis

The values of the constants appearing in these material models and EOS are those often presented in the technical literature.

Because the aim of this numerical study is only to put in evidence the cavitation phenomenon and for saving of the computer time, the water domain modelled by free particles was chosen only $20 \mathrm{~m}$ (Figure 8).

\section{Results and Discussions}

The present of the cavitation in a water point (on a particle) is demonstrated by the pressure evolution in that point, which include that negative pressure, usually between zero and $0.025 \mathrm{MPa}$. Here are some post-procesing pictures which show the pressure time evolution in some water points (particles), chosen inside and outside the bulk cavitation area.

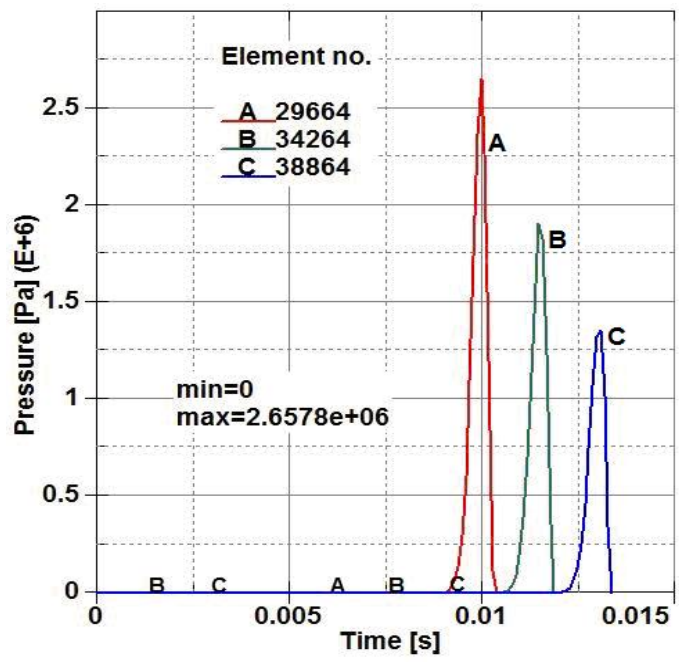

Fig. 9 Pressure evolution on particles outside the cavitation area

The Figure 9 presents the pressure evolution in time for three particles having the same depth $(1.825 \mathrm{~m})$ but with different distances $x$ towards the explosive (14.825, 17125 respectively $19.425 \mathrm{~m}$ ).

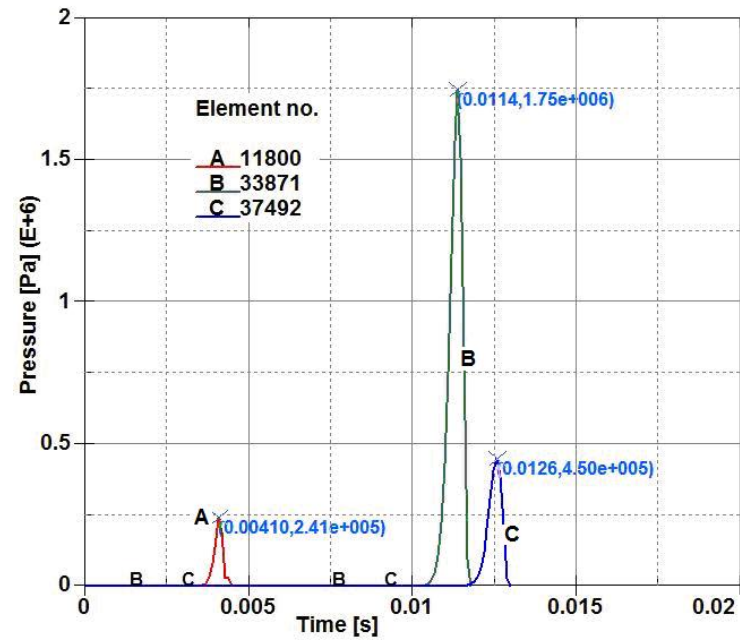

Fig. 10 Pressure evolution on particles inside the cavitation area

The Figure 10 shows the pressure time evolution for three particles chosen inside the cavitation domain. The $x$ coordinates of the particles are $5.875,16.925$ respectively 18.725 meters. The depth of the particles are: $0.025,1.475$ respectively 0.425 meters.

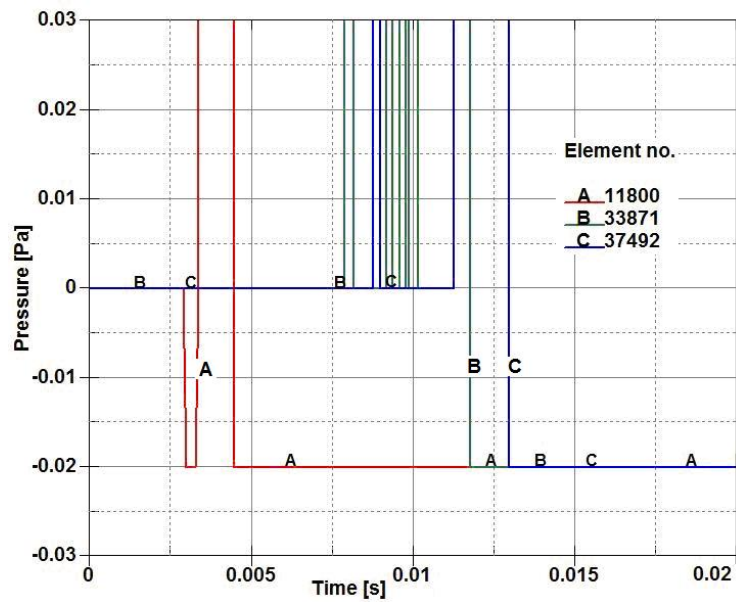

Fig. 11 Details of the lower pressure in the chosen particles

In the Figure 11 a detail regarding the lower pressure on the chosen particles is presented, where we can see that negative pressure, a characteristic of the cavitation phenomenon.

In the Figure 11, we can see that the 
cavitation process can be (particle 11800) the first phenomenon, comparatively with the increasing of the pressure, produced by the explosion wave propagation.

Also, we can see (particle 33871) that the cavitation appears at a moment, after some oscilation of the pressure.

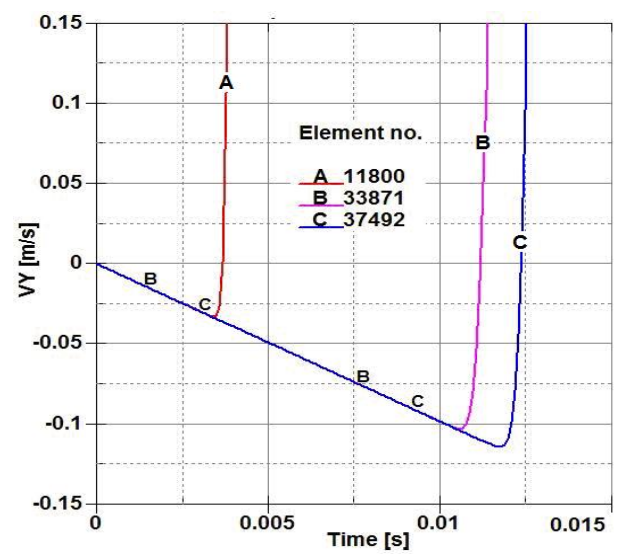

Fig. 12 Detail of the particle velocities

The present of the cavitation in those points in water, represented by particles, is also confirmed by the $y$-velocities of the particles, presented in the Figure 12.
The displacements and the velocities in the $y$-axis negative sense are the result of the reflected pressure which produces the cavitation phenomenon.

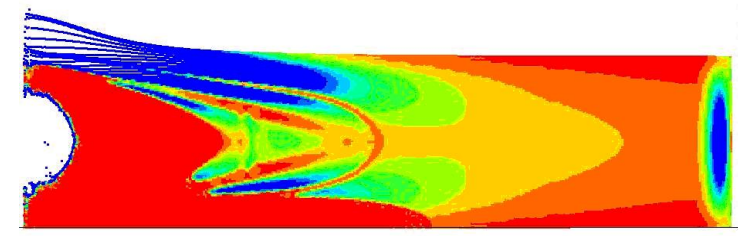

Fig. 13 Water density field

The water density field presented in the Figure 13 shows the present of cavitation (density values between 950 and 1000, under standard value).

\section{Conclusions}

Bulk cavitation phenomenon is present in all underwater explosions. Its effects upon structures belong to non contact explosion type. The study of bulk cavitation is useful and necessary because this cavitation type can cause direct damages and/or an amplification of damages. For all studies regarding underwater explosion, $\mathrm{SPH}$ method seems to be the best method.

\section{References}

[1] Andrzej Grzadziela, Ship Impact Modeling of Underwater Explosion, Journal of KONES Powertrain and Transport, Vol. 18, No. 2 2011, Poland.

[2] Arons, A.B., et al, Long Range Shock Propagation in Underwater Explosion Phenomena II, Underwater Explosion Compendium, Vol. 1, October 1949.

[3] Cole, R. H., Underwater explosions, Princeton University Press, 1948.

[4] Henry Tan, Underwater explosion (Part I), http://homepages.abdn.ac.uk./h.tan/pages/ teaching/explosion-engineering/Underwater-I.pdf

[5] Henry Tan, Underwater explosion (Part I), http://homepages.abdn.ac.uk./h.tan/pages/ teaching/explosion-engineering/Underwater-I.pdf

[6] Liu, G. R., Meshfree Methods, Mooving Beyond the Finite Element Method, Second Edition, CRC Press Taylor \& Francis Group, 2010, LLC, ISBN 978-1-4200-8209-8.

[7] Liu, M. B., Liu, G. R., Smoothed Particle Hydrodynamics (SPH): an Overview and Recent Developments, CIMNE, Barcelona, Spain 2010, Arch Comput Methods Eng (2010) 17: 25-76, DOI 10.1007/s11831-010-9040-7.

[8] Năstăsescu, V., Bârsan, Gh., Metoda particulelor libere în analiza numerică a mediilor continue, Editura AGIR, Bucureşti, 2015, ISBN 978-973-720-617-6.

[9] Webster G. Keith, Investigation of Close Proximity Underwater Explosion Effects on a Ship-Like Structure Using the Multi-Material Arbitrary Lagrangian Eulerian Finite Element Method, Master of Science in Ocean Engineering Thesis, Virginia Polytechnic Institute and State University, Blacksburg, 2007 